\title{
A combined viscosity-restricted intramolecular motion and mitochondrial targeting leads to selective tumor visualization
}

Received 00th January 20xx, Accepted 00th January 20xx

\author{
Le Yu, ${ }^{\ddagger a b}$ Jun Feng Zhang, ${ }^{\ddagger c}$ Mingle Li, ${ }^{\text {b }}$ Ying Zhou, ${ }^{* a}$ Peter Verwilst, ${ }^{* d}$ and Jong Seung Kim*b
}

DOI: $10.1039 / \times 0 x \times 00000 x$

\begin{abstract}
We report a novel fluorescent molecular conjugate V-M1 enabling an accurate visualization of tumor tissues. The emission wavelength of V-M1 exceeds $650 \mathrm{~nm}$, well within the near-infrared therapeutic window. Tumor accumulation of this cationic dye allows the visualization of cancerous cells as a function of mitochondrial viscosity.
\end{abstract}

Surgical resection of tumorous tissues has remained the most effective method for cancer treatment. During the procedure, the success of the surgery greatly depends on the completeness of the tumor removal. Incomplete tumor resection will lead to tumor recurrence, causing further damage. ${ }^{1}$ Therefore, the accurate visualization of tumor tissue plays a crucial role during an operation. ${ }^{2}$ However, the majority of conventional imaging methods, such as magnetic resonance $(M R)^{3}$ and ultrasound (US) ${ }^{4}$ are still associated with an unsatisfactory resolution, which translates into poor control over the precision of distinguishing tumor tissue from normal tissue. ${ }^{3}$ Imaging-guided surgery using fluorescence has a comparatively better imaging resolution and has the potential for a broad application in a clinical setting. ${ }^{5}$ However, the success of the method is determined by the tissue selectivity of the fluorescent dye, as well as the orthogonality of the fluorescence with tissue autofluorescence. ${ }^{6}$ This latter condition can be met by using near-infrared fluorescence covering the first therapeutic window (650-900 nm), where both absorbance, light scattering and autofluorescence are low. ${ }^{7}$

Viscosity, as the single-most important factor in diffusioncontrol processes, plays a vital role in chemistry and biological

a. College of Chemical Science and Technology, Yunnan University, Kunming 650091 P.R. China.

b. Department of Chemistry, Korea University, Seoul 02841, Korea

c. College of Chemistry and Chemical Engineering, Yunnan Normal University, Kunming 650500 , P. R. China

d. KU Leuven, Rega Institute for Medical Research, Medicinal Chemistry, 3000 Leuven, Belgium.

₹ These authors contributed equally.

$\uparrow$ Electronic Supplementary Information (ESI) available: $\left[{ }^{1} \mathrm{H},{ }^{13} \mathrm{C} N M R\right.$ and MS analyses of new compounds, experimental procedures, supplementary figures]. See DOI: $10.1039 / \times 0 \times x 00000 x$ activities. ${ }^{8}$ In fact, unusual variation in micro-viscosity of cell is associated with many human-diseases, such as diabetes, Alzheimer's disease, atherosclerosis and especially, cell malignancy. ${ }^{9}$ In general, compared to the vast majority of normal cells, the relative micro-viscosity in tumor cells is higher, which can be attributed to the accumulation of lactic acid in tumors. ${ }^{10}$ Thus, imaging cellular viscosity could represent an elegant solution to achieve the purpose of visualizing the tumor tissue. However, the large viscosity of cell membranes, particularly those bearing large amounts of glycoproteins or mucous cells, would likely represent major sources of interference. ${ }^{11}$

In order to overcome all the aforementioned challenges, herein, we report a novel viscosity-sensitive fluorescence molecule V-M1, which contains a positive charge (Scheme 1). As we will demonstrate below, this positive charge, in combination with the overall lipophilic nature of the dye, bestows a mitochondrial selectivity to the molecular conjugate. ${ }^{12}$ In view of the significantly higher mitochondrial membrane potential of cancerous tissues, ${ }^{13}$ this not only bypasses potential issues with cell membrane viscosity, but inherently increases the selectivity towards malignant tissues compared to other viscosity probes.

V-M1 was synthesized through a condensation reaction between a 1,8-naphthalimide derivative and 4-(diethyl amino)salicylic aldehyde. Through this ring-forming reaction, the intramolecular conjugated system is expanded, causing the maximum emission wavelength of V-M1 shift to $650 \mathrm{~nm}$, with a broad tail extending in the NIR region. The conjugation of the

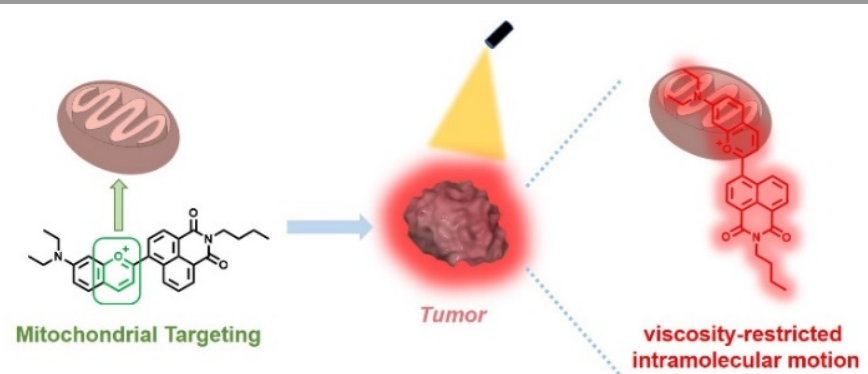

Scheme 1 Structure and mechanism of V-M1. 
(a)

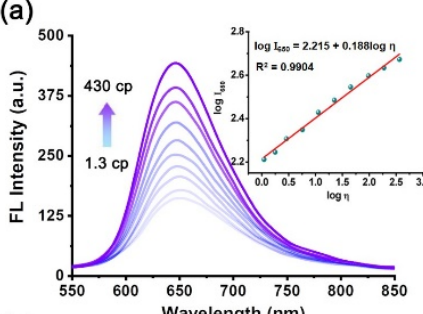

(c)

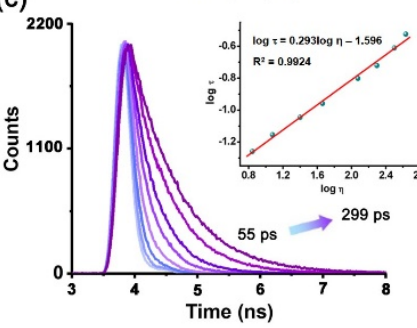

(b)

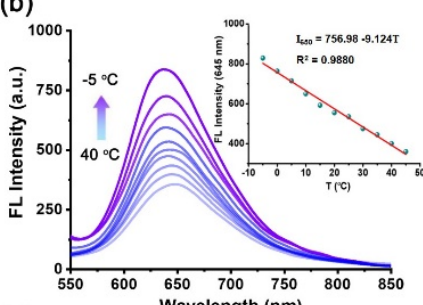

(d)

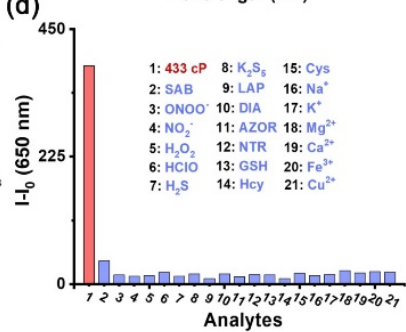

Fig. 1 (a) Fluorescence spectra of V-M1 $(10 \mu \mathrm{M}$, excited at $505 \mathrm{~nm})$ in mixed solvents with different proportions of methanol and glycerol at $25^{\circ} \mathrm{C}$. Inset: The linearity of the relationship between the fluorescence intensity and viscosity in mixed solvents, $R^{2}=$ 0.9904. (b) Fluorescence spectra and ratio of V-M1 $(10 \mu \mathrm{M}$, excited at $505 \mathrm{~nm})$ at different temperatures in methanol-glycerol $(3 / 7, v / v)$. Inset: The linearity of the relationship of the fluorescence intensity and the solvent temperature, $R^{2}=0.9879$. (c) Fluorescence lifetime spectra of V-M1 $(10 \mu \mathrm{M})$ in methanol/glycerol mixed solvents (excited at $470 \mathrm{~nm}$ ) at $25^{\circ} \mathrm{C}$. Inset: Linear relationship of $\log \tau$ and $\log \eta, R^{2}=0.9924$. (d) Fluorescent response of V-M1 $(10 \mu \mathrm{M}$, excited at $505 \mathrm{~nm})$ in the presence of various analytes $\left(50 \mu \mathrm{M}, 2 \mathrm{mg} / \mathrm{mL}\right.$ of serum albumin) at $25^{\circ} \mathrm{C}$.

two sub-components of the dye were effectuated through a bond with a predominant single bond character to enable intramolecular motion as a deexcitation pathway. The synthesis is shown in Scheme S1 (ESI+). The chemical structure of V-M1 was confirmed by ${ }^{1} \mathrm{H}$ NMR, ${ }^{13} \mathrm{C}$ NMR spectroscopy and mass spectrometry. The NMR and Mass spectra of V-M1 are shown in the Electronic Supporting Information (Fig S1-S6, ESI+).

The fluorescence of V-M1 demonstrated relatively minor changes in several solvents with different polarities, but an obvious fluorescence enhancement was observed in glycerol (Fig. S7, ESI ${ }^{+}$). To confirm this enhanced fluorescence was a result of the dramatically different viscosity, we performed two complementary experiments. Firstly, the viscosity-dependent response of $\mathbf{V}-\mathbf{M} \mathbf{1}$ was studied in media consisting of different ratios of methanol to glycerol. with viscosities ranging from 1.3 CP to $430 \mathrm{cP}$ (Table. S1, ESI+). As show in Fig. 1a, V-M1 exhibits a weak emission intensity at $650 \mathrm{~nm}$ in solvents with low viscosities. However, the mission intensity of V-M1 is notably enhanced as a result of the increased viscosity of the solvent. A 3-fold enhancement in fluorescence intensity can be observed, with a good linear dependence between $\log I_{650}$ and $\log \eta$ (viscosity), evidenced by the correlation coefficient of 0.9904. In a second approach, the temperature of a methanol-glycerol $(3 / 7, v / v)$ was regulated between $45{ }^{\circ} \mathrm{C}$ and $-5{ }^{\circ} \mathrm{C}$, to cause temperature-dependent viscosity changes. ${ }^{14}$ The intensity of the emission peak at $650 \mathrm{~nm}$ clearly increased as the temperature decreased, and an excellent linear relationship exists between $I_{650}$ and the temperature $\left(R^{2}=0.9879\right)$. Very similar experimental results were obtained using ethanol and glycerol as components of the mixed solvent media (Fig. S8, $\mathrm{ESI}+$ ). Furthermore, with increasing viscosity, the absorbance of V-M1 in the mixed solvents with different proportions of

ethanol/glycerol and methanol/glycerol both exhibit regular enhancements at around $505 \mathrm{~nm}$ (Fig. S9 and S10, ESIt).

To exclude fluorescent responses from sources other than viscosity, we performed an experiment in which the viscosity was kept constant, but the polarity was varied. In different proportions of 1,4-dioxane/water as a solvent, only minor fluorescence changes were observed, as shown in Fig. S11 $(E S I+)$. The influence of the $\mathrm{pH}$ on the fluorescence was studied as well, and we further studied the pH stability of V-M1 in the water/glycerol system (4:6, v/v, $0.01 \mathrm{M}$ PBS buffer). The $\mathrm{pH}$ titration scatter diagram demonstrates that V-M1 exhibits a stable fluorescence intensity in the $\mathrm{pH}$ 3-8.5 range, indicating that $\mathbf{V - M 1}$ is capable of working at all $\mathrm{pH}$ levels commonly encountered in in vivo environments. (Fig. S12, ESIt). Finally, we tested if any common biologically relevant small molecules and commonly overexpressed enzymes could be excluded as potential sources of interference. The results are summarized in Fig. 1d. As can be seen potential interferents such as ONOO', $\mathrm{NO}_{2}{ }^{-}, \mathrm{H}_{2} \mathrm{O}_{2}, \mathrm{HClO}, \mathrm{H}_{2} \mathrm{~S}, \mathrm{~K}_{2} \mathrm{~S}_{5}$, leucine aminopeptidase (LAP), diaphorase (DIA), azo reductase (AZOR), nitroreductase (NTR), glutathione (GSH), Homocystine (Hcy), cysteine (Cys), some cations $\left(\mathrm{Na}^{+}, \mathrm{K}^{+}, \mathrm{Fe}^{3+}, \mathrm{Cu}^{2+}, \mathrm{Ca}^{2+}, \mathrm{Mg}^{2+}\right)$ and serum albumin (SAB) only produced negligible fluorescent changes for V-M1, as compared to the fluorescent response in a high viscous solvent mixture.

Prior to cell imaging, the cytotoxicity of V-M1 was tested in various cell lines, with $\mathrm{IC}_{50}$ values ranging from $9.65 \mu \mathrm{M}$ to 22.51 $\mu \mathrm{M}$ in cancerous cells and $>60 \mu \mathrm{M}$ in a normal cell line (Fig. S13,
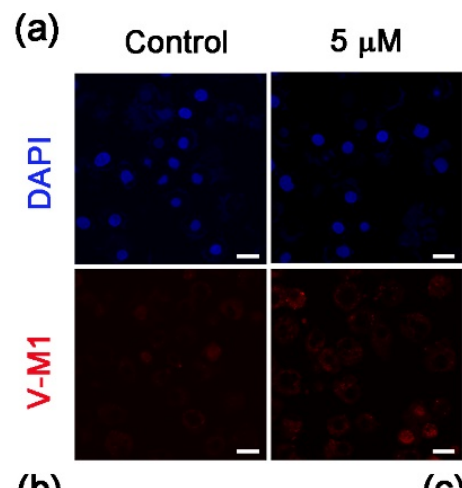

$10 \mu \mathrm{M}$ $15 \mu \mathrm{M}$

(b)
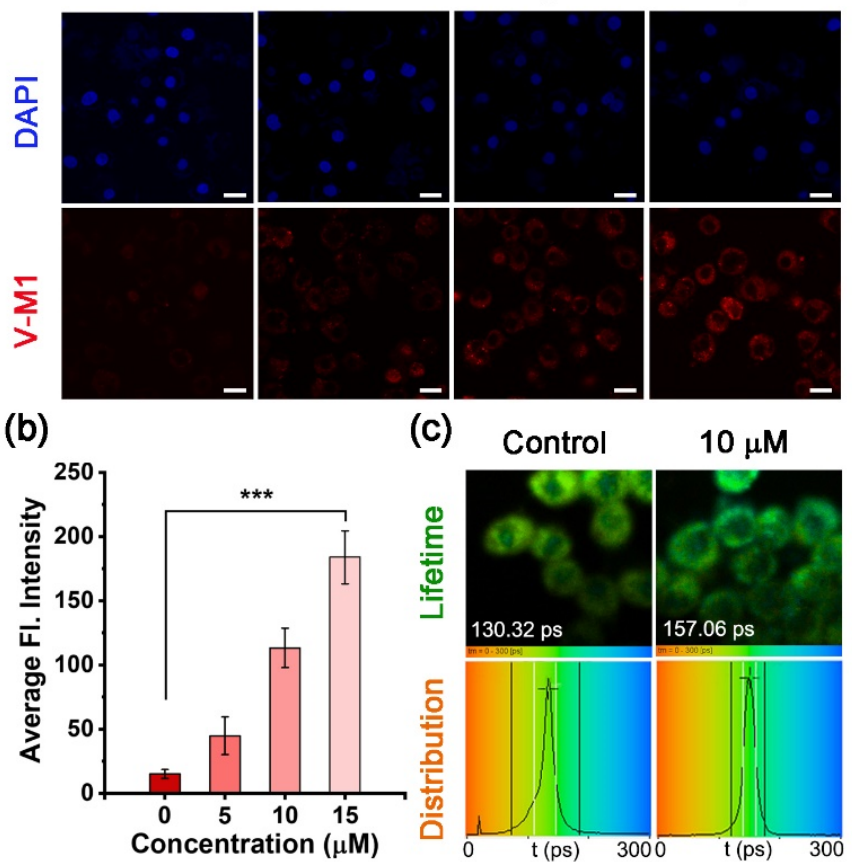

(c)

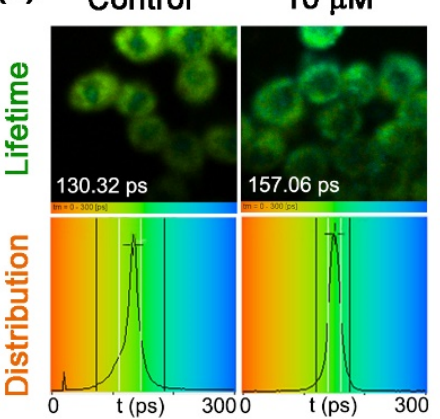

Fig. 2 (a) CLSM imaging of HeLa cells stained with V-M1 $(10 \mu \mathrm{M})$, DAPI (10 $\mu \mathrm{M})$ Cells were treated with different concentrations of monensin $(0 \mu \mathrm{M}, 5 \mu \mathrm{M}, 10 \mu \mathrm{M}$, $15 \mu \mathrm{M})$; (b) Average fluorescence intensity of each group of cells in panel (a), Bars denote the average of $n=3$ measurements of random cells, while error bars denote the standard deviation; (c) Fluorescence lifetime imaging of V-M1 in HeLa cell. Cells were imaged with and without the addition of monensin $(0$ and $10 \mu \mathrm{M})$. $* * * \mathrm{p}<0.001$ 
ESI + ). This differential toxicity clearly hints at an intracellular target important to cellular proliferation. In subsequent cell studies, the concentrations of V-M1 never exceeded $10 \mu \mathrm{M}$ to avoid significant toxicity induction. In order to utilize V-M1 as an efficient tool for the detection of intracellular viscosity, the intracellular location of $\mathbf{V}-\mathbf{M} \mathbf{1}$ was identified first. The colocalization results of V-M1 were obtained using a CLSM (Confocal laser scanning microscope) with Mito Tracker Green (MTG), a commercial green-fluorescence marker for mitochondria. As shown in Fig. S14 (ESIt), V-M1 shows a distribution consistent with that of MTG, and the intensity profile of linear regions of interest (ROIs) between V-M1 and MTG also tends to be consistent, supported by an excellent Pearson's coefficient of 0.95 . Furthermore, no interference from the cellular membrane was observed for V-M1.

Monensin, well-known for its ability to increasing the intracellular viscosity by causing structural changes or swelling of mitochondria, ${ }^{15}$ was used to study the ability of V-M1 to respond to cellular viscosity changes. Upon treatment with monensin, a distinct red fluorescence of V-M1 can clearly be observed in HeLa cells (Fig. 2a and S15, ESI ${ }^{+}$). Notably, as shown in Fig. $2 \mathrm{~b}$, with the concentration of monensin increasing, the average fluorescence intensity is enhanced proportionally. All these observations point to V-M1 as an effective fluorescent molecular tool for detecting viscosity fluctuations in HeLa cells.

To further confirm the changes in fluorescence as reported in Fig. $2 \mathrm{a}$ and $\mathrm{b}$ are caused by the changes in the viscosity, cells treated under similar conditions were subjected to fluorescence lifetime imaging. As shown in Fig. $2 \mathrm{c}$ and S16 (ESIt), the average fluorescence lifetime of V-M1 in HeLa cells was measured as $130.32 \mathrm{ps}$, which indicates the viscosity of $64.6 \mathrm{cP}$ on the basis of the linear relationship in Fig. 1c. Upon treatment with monensin $(10 \mu \mathrm{M})$, a clear enhancement of the fluorescence lifetime can be observed. The average fluorescence lifetime in HeLa cells increases to 157.06 ps, which corresponds to a viscosity estimate of $104.0 \mathrm{cP}$. Importantly, these data correspond well with a previously reported normal mitochondrial viscosity of $63.5 \mathrm{cP}$ and abnormal viscosity of 91.9 in HeLa cells, ${ }^{16}$ thus validating V-M1 as a quantitative imaging agent for intracellular viscosity. (a)

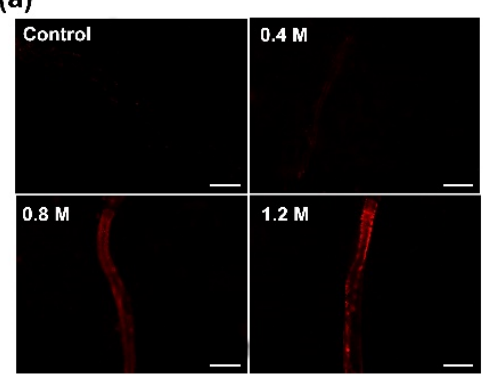

(b)

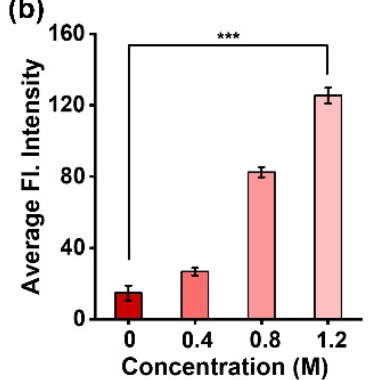

Fig. 3 (a) Fluorescence imaging of $C$. elegans stained with V-M1, in nematodes subjected to increasing concentrations of $\mathrm{NaN}_{3}$. Images were obtained using an excitation wavelength of $505 \mathrm{~nm}$, with the emission being monitored in a spectral window encompassing 600-650 nm. Scale bar: $20 \mu \mathrm{m}$; (b) Average fluorescence intensity of each group from panel (a). Bars denote the average of $n=3$ measurements of similar regions of interest from the different part of the same nematode, while error bars denote the standard deviation. ${ }^{* * *} p<0.001$.
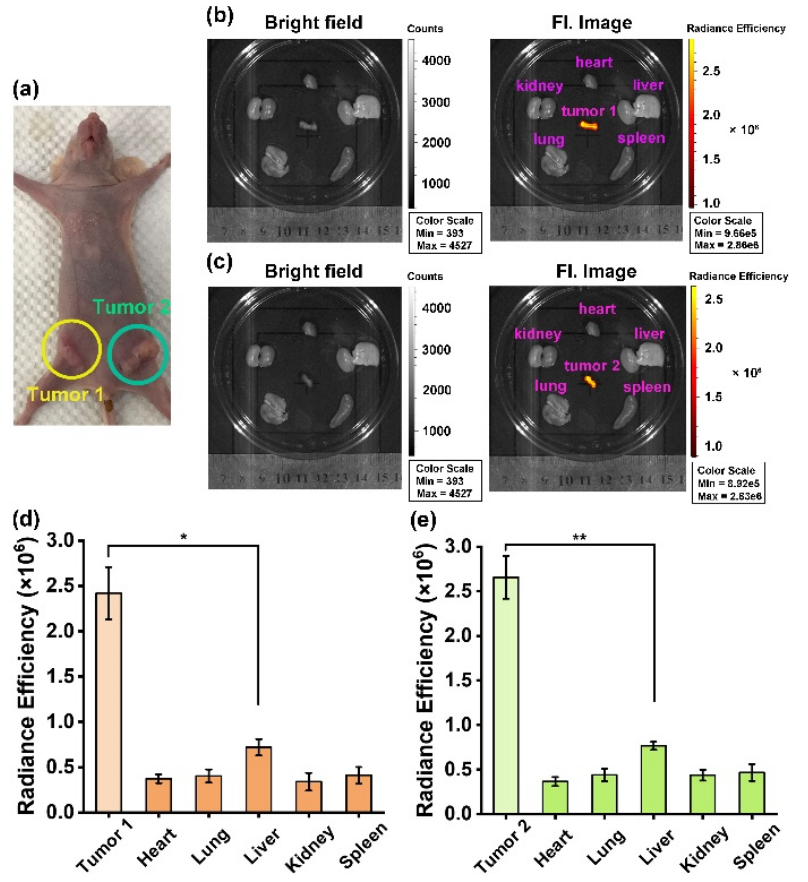

Fig. 4 (a) A photo of a mouse used in this experiment, and tumors are shown in the circles; (b) Bright field and fluorescence signal imaging of the 4T1 xenographt tumor 1; (c) Bright field and fluorescence signal imaging of the 4T1 xenographt tumor 2. All organs and tumors were incubated with $100 \mu \mathrm{M}$ of V-M1 in the ethanol-PBS system $(1: 10, v / v)$ for $4 \mathrm{~h}$ at $37^{\circ} \mathrm{C}$. Images were obtained using an excitation wavelength of $530 \mathrm{~nm}$, with the emission being monitored in the 600$650 \mathrm{~nm}$ spectral window. (d) The radiance efficiency of the tumor 1 compared to other organs; (e) The radiance efficiency of the tumor 2 compared to other organs. Bars denote the average of $n=3$ measurements of similar regions of interest from the same tumors or organs, while error bars denote the standard deviation. ${ }^{*} p<$ $0.05, * * p<0.01$

As outlined above, we characterized V-M1 as an accurate detection tool for viscosity at the cellular level. However, the microenvironment of living animals is complex. Thus, we chose C. elegans, a widely used biological model of invertebrates, to conduct fluorescence imaging. $\mathrm{NaN}_{3}$ is an anesthetic for $C$. elegans, resulting in different degrees of physiological disfunction, ${ }^{17}$ associated with abnormal viscosity levels. As show in Fig. 3 and $\mathrm{S} 17$ (ESI $)$ ), the images of $C$. elegans belonging to the control group hardly showed any fluorescent emission. However, with the concentration of $\mathrm{NaN}_{3}$ increasing, a strongly increased red fluorescence was observed, meaning that V-M1 is capable of detecting the viscosity changes in vivo in multicellular animals.

Based on experimental data, we anticipate the potential of $\mathbf{V}$ M1 for tumor visualization through its higher viscosity, combined with mitochondrial targeting. Therefore, the fluorescence signal imaging for visualization in mouse xenograft model for tumor selective targeting and biodistribution was conducted. We established 4T1 murine mammary tumorbearing BALb/c mice model. When the tumors grew up to the experiment experiment-required size (8-12 $\mathrm{mm}$ in diameter), mice were euthanized using the cervical dislocation method, then the tumor and organs were separated from the mice with a scalpel and divided into two groups, one group was placed on glass-bottom culture dishes, incubated with $100 \mu \mathrm{M}$ V-M1 in ethanol-PBS $(0.01 \mathrm{M}, \mathrm{pH}=7.4)(1: 10, v / v)$ under normoxia 
conditions at $37{ }^{\circ} \mathrm{C}$ for $4 \mathrm{~h}$, the other was incubated with ethanol-PBS $(0.01 \mathrm{M}, \mathrm{pH}=7.4)(1: 10, v / v)$ under normoxia at 37 ${ }^{\circ} \mathrm{C}$ for $4 \mathrm{~h}$. Tumors and organs were washed with PBS before imaging.

As shown in Fig. 4, it is obvious that both tumor 1 and 2 show a strong fluorescence-signal emission after being treated with V-M1. By contrast, other vital organs such as the lungs, heart, spleen and kidneys hardly show any fluorescence (Fig. 4b, c). Importantly, the liver, which usually interferes with tumorspecific detection, also exhibits a very weak fluorescent signal. As a result of this significant signal intensity difference between the liver and tumor (Fig. 4d, e), V-M1 would be very appealing for tumor imaging. In a control experiment (Fig. S18, ESI+), no fluorescent signal can be detected without the addition of $\mathbf{V}$ M1, demonstrating a clear lack of autofluorescence that may exist in the tumor under these imaging conditions. This phenomenon could be attributed to the inclusion of a cation in the structure of V-M1.

Some cationic-molecules can be quickly internalized by cancer cells through the adsorption-mediated transcytosis (AMT), then those molecules would be intracellularly shifted and exocytosed into the interstitium and finally internalized by the surrounding cells and transferred to cells at the lower layers. Thus, those cationic molecules can penetrate deeper into the tumor tissues. ${ }^{18}$ In this way, the cationic V-M1 maybe undergoes both endocytosis and transcytosis, which enabling both transendothelial and transcellular transport. As a result, VM1 can be uniformly distributed throughout the tumor and respond to the abnormal viscosity (Fig. $4 \mathrm{~b}, \mathrm{c}$ ). The fluorescence signal imaging of slices of tumor 1 and 3 (Fig. S19) further supported this assumption.

Although the fluorescence signal of a slice of tumor 1 is relatively weak, it still demonstrated that a small portion of $\mathbf{V}$ M1 transfers deep into the tumor, compared to other organs, and this result was consistent with our assumption. In addition, there was no fluorescence signal could be detected in the control group, ruling out tissue auto-fluorescence as a potential source of interference. All these results indicate that V-M1 has a capacity to penetrate the tumor through AMT and specifically mark the tumor by sensing its abnormal viscosity.

In conclusion, V-M1, a novel fluorescence probe for the visualization of tumor through monitoring viscosity was presented. Spectral analysis, fluorescence and fluorescence lifetime imaging of HeLa cells provided a clear evidence for a favorable biocompatibility and a quantitative measurement of the micro-viscosity. The fluorescence imaging experiments with C. elegans indicated that V-M1 has a capacity to detect the fluctuation of viscosity in living multicellular animals. Notably, during the ex vivo biodistribution imaging test, 4T1 xenographt tumors were specifically identified among different tissues by V-M1 fluorescence, demonstrating a potential for guiding surgical resection of tumor through detecting an increased viscosity, combined with mitochondrial targeting. All these results suggest that $\mathbf{V}-\mathbf{M} \mathbf{1}$ can be utilized as an efficient tool for tumor visualization.

This work was supported by CRI project no. 2018R1A3B1052702 (J.S.K.) of National Research Foundation
(NRF) grant funded by Korea government. Zhou and Zhang thank the support by the National Natural Science Foundation of China (Nos. 21672185 and 21867019).

\section{Conflicts of interest}

There are no conflicts to declare.

\section{Notes and references}

1 (a) J. M. Sarmiento, G. Heywood, J. Rubin, D. M. Ilstrup, D. M. Nagorney and F. G. Que, J. Am. Coll. Surgeons, 2003, 197, 29.

2 Y. Park, Y. -M. Ryu, T. Wang, Y. Jung, S. Kim, S. Hwang, J. Park, D.-J. Bae, J. Kim, H. Moon, H.-S. Lim, S.-Y. Kim, E. Chung, K. H. Kim, S. Kim and S.-J. Myung, Adv. Funct. Mater., 2018, 28, 1703450.

3 K. Yang, L. Hu, X. Ma, S. Ye, L. Cheng, X. Shi, C. Li, Y. Li and Z. Liu, Adv. Mater., 2012, 24, 1868.

4 S. Mallidi, G. P. Luke, and S. Emelianov, Trends Biotechnol., 2011, 29, 213.

5 C. W. Chi, Y. Du, J. Z. Ye, D. Q. Kou, J. D. Qiu, J. D. Wang, J. Tian and X. Y. Chen, Theranostics, 2014, 4, 1072.

6 Y. Rivenson, H. D. Wang, Z. S. Wei, K. D. Haan, Y. B. Zhang, Y. C. Wu , H. Günaydın, J. E. Zuckerman, T. Chong, A. E. Sisk, L. M. Westbrook, W. D. Wallace and A. Ozcan, Nat. Biomed. Eng., 2019, 3, 466.

7 H. D. Li, Q. C. Yao, W. Sun, K. Shao, Y. Lu, J. Chung, D. Kim, J. L. Fan, S. Long, J. J. Du, Y. Q. Li, J. Y. Wang, J. Yoon, and X. J. Peng, J. Am. Chem. Soc., 2020, 142, 6381.

8 K. Luby-Phelps, Int. Rev. Cytol., 2000, 192, 189.

9 (a) P. M. Moriarty and C. A. Gibson, Cardiovasc. Rev. Rep., 2003, 24, 321; (b) G. Deliconstantinos, V. Villiotou and J. C. Stavrides, Biochem. Pharmacol., 1995, 49, 1589; (c) S. J. Singer and G. L. Nicolson, Science, 1972, 175, 720.

10 (a) M. V. Guyer, Cancer Res., 1942, 2, 16; (b) M. V. Guyer, Anat. Rec., 1947, 73, 17.

11 (a) N. Gupta, S. I. Reja, V. Bhalla, M. Gupta, G. Kaur and M. Kumar, Chem. Commun., 2015, 51, 10875; (b) F. Guo, W. P. Gai, Y. Hong, B. Z. Tang, J. Qin and Y. Tang, Chem. Commun., 2015, 51, 17257.

12 (a) T. B. Ren, W. Xu, F. P. Jin, D. Cheng, L. L. Zhang, L. Yuan, and X. B. Zhang, Anal. Chem., 2017, 89, 11427; (b) J. F. Zhang, L. E. Guo, T. N. Zang, Y. L. Duan, X. Y. Liu, Z. G. Yang, P. Verwilst, K. J. Luo, G. K. Wang, J. F. Kou, Y. Zhou, and J. S. Kim, Chem. Asian J., 2015, 10, 1165.

13 S. Davis, M. J. Weiss, J. R. Wong, T. J. Lampidis, L. B. Chen, J. Biol. Chem., 1985. 260, 13844.

14 X. J. Peng, Z. G. Yang, J. Y. Wang, J. L. Fan, Y. X. He, F. L. Song, B. S. Wang, S. G. Sun, J. L. Qu, J. Qi, and M. Yan, J. Am. Chem. Soc., 2011, 133, 6626.

15 (a) S. P. Soltoff and L. J. Mandel, J. Membr. Biol., 1986, 94, 153; (b) M. J. Hansson, S. Morota, M. Teilum, G. Mattiasson, H. Uchino and E. Elmér, J. Biol. Chem., 2010, 285, 741.

16 A. Jiménez-Sánchez, E. K. Lei, and S. O. Kelley, Angew. Chem. Int. Ed., 2018, 57, 8891.

17 (a) R. M. Michelle, M. L. Elizabeth, D. B. Kristy, E. S. Karen and E. W. Glenn, Cell Stress Chaperones, 2003, 8, 1; (b) F. Y. Christopher, V. G. Christopher, D. T. S. Aravinthan and I. B. Cornelia, Methods Cell Biol., 2012, 107, 177.

18 (a) Q. Zhou, S. Q. Shao, J. Q. Wang, C. H. Xu, J. J. Xiang, Y. Piao, Z. X. Zhou, Q. X. Yu, J. B. Tang, X. R. Liu, Z. H. Gan, R. Mo, Z. Gu and Y. Q. Shen. Nat. Nanotechnol., 2019, 14, 799; (b) H. Hyun, M. H. Park, E. A. Owens, H. Wada, M. Henary, H. J. M. Handgraaf, A. L. Vahrmeijer, J. V. Frangioni and H. S. Choi, Nat. Med., 2015, 21, 192. 